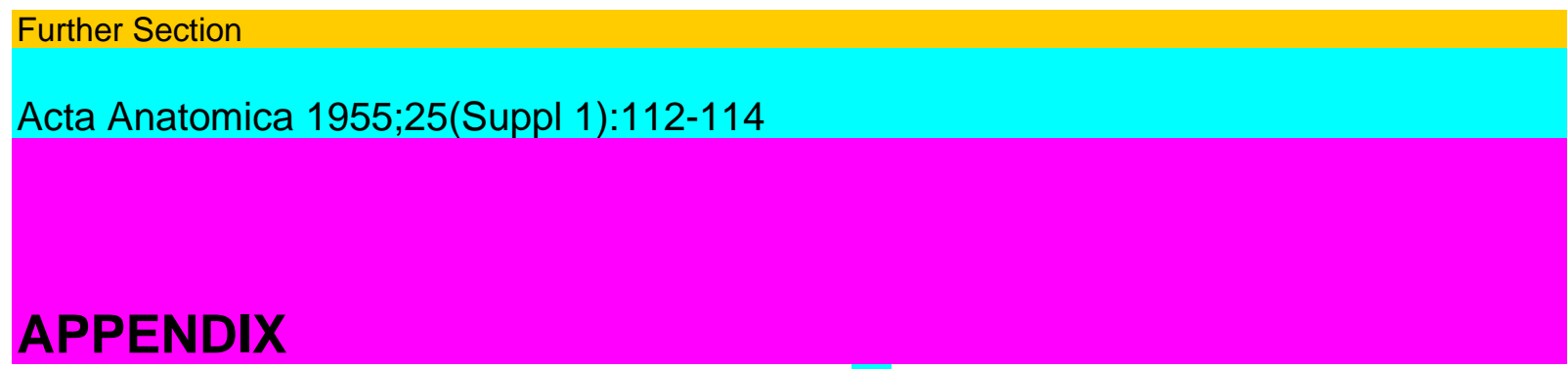

Method Used in Making Positive Prints in the Size 24x36 mm from »Leica« Negatives.

by

\title{
EINAR HANSEN
}

In making the positives presented in this monograph, the ordinary equipment for contact printing from »Leica « negatives could not be used, as this is designed for printing a series of positives in which the short sides of the individual prints adjoin. A series of, for example, seven positives made with this equipment would have a length of about $270 \mathrm{~mm}$, while the length would be only about $180 \mathrm{~mm}$, if a method was used by which the long sides of the prints were adjoined. Projection printing was therefore used, because this made it possible to place the printing-paper strip in such a manner that its longitudinal direction was at a right angle to that of the »Leica« negatives. Printing paper of a width of $40 \mathrm{~mm}$ was required to accomodate the 36-mm height of the negatives, and as this was not available in a standard size, sheets of printing paper of the size $180 \times 240 \mathrm{~mm}$ were used. Each sheet was cut into six strips, measuring $40 \times 180 \mathrm{~mm}$. During the printing process, such a strip was placed in a specially made printing frame with an adjustable feed device, which moved the paper strip so much that it just corresponded to the width of a Leica negative plus a suitable interspace. The enlarger used was a Leitz Focomat model. However, as a projection of the negative in the size 1:1 could not be obtained with the usual Leitz Elmar objective having a focal length of $50 \mathrm{~mm}$, this had to be replaced by a $90 \mathrm{~mm}$ Leitz Elmar objective modified in such a manner that its normal mounting was extended with the extension rings marked M. 1:1, M. 1:15 and B. from the Leitz Universal reproduction set. As the mains voltage, which is nominally 220 volts, is not by far constant enough for the present purpose, and as the 75-watt opal-glass lamp of the enlarger, when fed by a nominal voltage of 220 volts, actually burns at an excess voltage, the luminous intensity will vary about 30 per cent in the absence of compensating measures. The enlarger was therefore connected to the mains supply in series with a rheostat, max. $110 \mathrm{ohms}$, and a voltmeter was connected in parallel with the lamp. In this way it was possible to check the voltage across the lamp and, by means of the rheostat, to compensate variations in the mains voltage, as such variations were of relatively slow onset. Experiments showed that the relation between the voltage across the lamp and its luminous intensity was linear in the range from 205 to 240 volts. The printing was made at 210 volts across the lamp. When the enlarger had been adjusted to projection in the ratio of $1: 1$ with fully opened objective aperture,

113

the intensity of the lamp was measured as 10 foot-candles with a General Electric photo-electric meter, Type DW-58 (without hood), placed on the baseboard of the enlarger.

As the entire series of negatives were made under standardized conditions, attempts were first made to use a standardized printing process in the treatment of all the negatives, irrespective of the degree of blackening. Preliminary printing tests made with the densest and lightest negative in the series seemed to show that this was possible. The printing paper used was Agfa 2 silverbromide paper, extra soft, which was processed in a soft-working metol developing solution in a 
dilution of 1:5 in order to obtain the longest possible scale of blackening. This developing solution (Agfa No. 105) is composed as follows: -

Water $1000 \mathrm{ml}$.

Metol $15 \mathrm{~g}$

Sodium sulphite, desiccated 75 -

Potassium carbonate 75 -

Potassium bromide 2 -

On the basis of exposure tests carried out at the aforementioned luminous intensity with subsequent development as just described, the exposure time was fixed at 10 seconds. The exposure time was measured by a Hirsch print timer inserted in the lamp circuit of the enlarger. The circuit was closed by depressing the push-button switch of the timer and opened by the timer at the end of the pre-set interval. In order to obtain a uniform degree of development, it would have been desirable to process all the printing-paper strips at the same time in the same batch of developing solution, but due to the relatively large number of strips this proved impossible, for which reason another method had to be adopted. By finishing off each series of positives with a uniformly blackened control field it should be possible to develop each series separately and yet obtain practically the same degree of blackening of the control fields and, hence, standardized conditions of development for all the positives, so that their variations in the blackening paralleled those in the staining intensity in the tissue sections. The control field was made as follows. After having made the prints which were to be on the paper strip, the film was withdrawn from the enlarger, and the intensity of the enlarger lamp was appropriately diminished by placing a neutral-grey filter on the printing frame, before the light rays reached the paper strip. The same end might have been obtained by a reduction of the exposure time and removal of the grey filter, but the former method was preferred because it was then possible to leave the adjustment of the print timer intact. The grey filter consisted of a Chance Brothers neutral-glass filter No. ON. $\cap$ superimposed on a Chance Brothers neutral-glass filter No. ON . . For this combination, the transmission percentage was measured as 1.5 per cent on a Photovolt transmission densitometer, model 500-A. The developing time was $23 / 8$ minutes, and at the end of this time the paper strip was placed in a short-stop bath of dilute acetic acid, and then in turn transferred to the fixing bath, a weak soda solution for neutralization of acidity, and to the rinsing bath, and dried on a high-gloss ferrotype press. After drying, the degree of black-

\section{4}

ening of the control field was measured on the reflection-density unit of the aforementioned densitometer as 0.9. In order to interrupt the development in the yellow-green light in the darkroom, when the density of the control field, 0.9 , was reached, the control field was compared with another paper strip of the same density. Such a reference paper strip was made by exposing seven fields for 10 seconds through the grey filter; developing time i $\backslash$ minutes.

After these procedures, prints were made from the entire series of negatives. From each film strip, which represented one experimental animal, five prints were made in order to obtain five uniform series of positives. When prints had been made from all the films, the paper strips were developed in a tray which was so large that five strips could be placed side by side. The reference strip was held in the left hand, while all necessary manipulations were made with the right hand. The five strips were placed in the developing solution one by one, and therefore also reached the proper degree of blackening one by one; there was even sufficient time for holding each strip beside the reference strip at the decisive moment. 
However, it proved to be impossible to obtain satisfactory positives of the entire series of negatives; some were too dark with loss of detail and therefore unsuited for reproduction. New prints were therefore made from this part of the series under the same conditions, yet with the modification that the intensity of the enlarger lamp was reduced to 2 foot-candles by stopping down the aperture of the objective. The blackening of the control field was then reduced by the same ratio as the light intensity (density 0.18 ). A new reference strip with this value was made, but instead of seven uniform fields as on the dark reference strip, the fields of the second reference strip were exposed for 4, 5, 6, 7, 8, 9 and 10 seconds, respectively, through the grey filter, whereas the developing time was the same as for the dark reference strip. In this way a series of fields with slightly increasing blackening was obtained, with a density of 0.18 in the centre. The purpose was that as the density of the control field increased, this field should be shifted along the reference strip until a density of 0.18 was reached, at which time developing should be interrupted. It appeared that a greater degree of accuracy was obtained in this way, probably because it seemed easier to determine when the density of the control field was greater or smaller than the two fields adjacent to the reference field having a density of 0.18 . Subsequent densitometric measurements on the control fields of the latter part of the series revealed a maximum deviation of 5 per cent. Densitometric measurements on the dark and light control fields showed good agreement, the difference in density being as 5:1, corresponding to a luminous intensity of 10 foot-candles for the dark and 2 foot-candles for the light control fields. 